\title{
Managing Urban Waste Collection through Timed Automata Based Fuzzy Cognitive Maps
}

\author{
Giovanni Acampora ${ }^{1}$, Vincenzo Loia ${ }^{2}$, and Autilia Vitiello ${ }^{2}$ \\ 1 School of Industrial Engineering, Information Systems, \\ Eindhoven University of Technology, \\ P.O. Box 513, 5600 MB, Eindhoven, The Netherlands \\ g. acampora@tue.nl \\ 2 Department of Computer Science, \\ University of Salerno, \\ Fisciano, Salerno, 84084, Italy \\ \{loia, avitiello\}@unisa.it
}

\begin{abstract}
In the last years, the increasing urbanization has constrained to face the dramatic growth of the urban waste production and the consequent socio-economical and environmental impact. The relevance of finding an optimal waste management further increases when it involves hazardous materials, since they represent a vulnerable infrastructure sector for homeland defense. Although there is a general agreement on the best strategies for solving urban garbage problem, an opportune waste management seems far due to its intrinsic complexity arising from necessity of dealing with several factors which are often in conflict each other. Over the years, several computerized waste management systems, including deterministic models and fuzzy approaches, have been developed aimed at addressing this complex problem. However, all these approaches do not consider relevant factors which could affect decision policies related to waste treatment, i.e., the rapid evolutions and modifications occurring in a complex scenario such as the urban environment. In order to overcome this drawback, this paper presents an innovative waste management simulation system based on a new timed cognitive inference engine, named Timed Automata based Fuzzy Cognitive Map (TAFCM). A TAFCM is able to simulate the dynamic features of a waste management environment thanks to its temporal benefits due to its ability of dealing with the concept of time in a direct way. As shown in the experimental section, TAFCMs represent a suitable and efficient methodology to manage the waste production problem.
\end{abstract}

Keywords: Urban Waste Management System, Computational Intelligence, Cognitive Modeling, Fuzzy Cognitive Maps, Timed Automata, Timed Automata based Fuzzy Cognitive Maps.

\section{Introduction}

In last years, urbanization has been one of the most strong and clear changes affecting all the world. The rapid and constant growth of urban population has led

G. Quirchmayr et al. (Eds.): CD-ARES 2012, LNCS 7465, pp. 501-515, 2012.

(C) IFIP International Federation for Information Processing 2012 
to a dramatic increase in urban waste production, with a crucial socio-economic and environmental impact [1]. The importance of finding an optimal waste management further increases when it involves hazardous garbage including any radioactive material manufactured, used, transported, or stored by industrial, medical, or commercial processes. Indeed, hazardous waste represents an environmental infrastructure sector vulnerable to attacks and, for this reason, its treatment goes into homeland defense missions. In the last years, legislation and regulations have been instituted in order to guide the management of waste, hazardous and not, and improve waste policies. Nowadays, there is a general agreement on the best strategies for sustainable management of urban waste which consists in minimising waste production and optimizing waste recovery. Indeed, it is proved that the recovery of the waste is an essential way to reduce the natural resource depletion and create alternative energy sources. Moreover, it is necessary to decrease the use of landfill sites which lead to damage human mental and physical health and represents a more suffered problem in big cities where there is no appropriate land filling zones.

However, an appropriate waste management seems far from being attained [1, due, above all, to the intrinsic complexity of the problem. Indeed, an optimal urban waste management involves various relative factors, which are often in conflict. The most important factors are mainly economic (e.g., system cost and system benefit), environmental (air emission, water pollution) and technological (the maturity of the technology). This complexity has led to the necessity of developing integrated, computerized systems for obtaining more generalized, optimal solutions for the management of urban waste collection [2]. In last years, there have been many solutions developed for the implementation of a waste management system which would monitor and manipulate the generated waste. The theoretical approaches in literature refer to issues concerning the conflict between urban residents and the municipality for the selection of sites for waste treatment, transshipment stations and disposal, or the issue of waste collection and transport as well as its impact to human health due to noise, traffic, etc [2]. Several deterministic models, including Linear Programming (LP) [3] 4] 5], have been exploited for optimizing sitting and routing aspects of waste collection networks. However, uncertainty often plays a prominent role in dealing waste management problems. Possible sources of uncertainty are the random nature of waste production and the vagueness in planning objectives and constraints. For this reason, fuzzy mathematical programming approaches [6] [7] 8] have been introduced in the waste collection management.

However, all these approaches do not consider relevant factors which could affect decision policies related to waste treatment, i.e., the rapid evolutions and modifications occurring in a complex scenario such as the urban environment. The aim of this paper is to present a waste management simulation system capable of supporting a municipal government in their dynamic decisions related to waste treatment and safe disposal. In detail, our proposal is aimed at simulating a waste management environment by using an extension of Fuzzy Cognitive Maps (FCMs), named Timed Automata based Fuzzy Cognitive Maps (TAFCMs) 
9. FCMs can be viewed as inference networks that use cyclic, directed graphs to represent the fuzzy causal relationships among fuzzy concepts. FCMs' inference engine is based on an easy, iterative and numeric process and, consequently, FCMs are able to represent knowledge and implement inference operators with greater flexibility as compared to many other available methods. Nevertheless, in spite of these benefits, conventional FCMs are not capable of dealing with the concept of time in a direct way. For this reason, a new timed cognitive inference engine, i.e., TAFCMs, has been introduced for improving FCMs' time representation of qualitative system dynamics. Thanks to this feature, TAFCMs represent a promising method for implementing a waste management simulation system where decisions to be made are usually dependent on the temporal events. The rest of the paper is devoted to present this new model and show how it is efficiently able to simulate a dynamic waste management environment.

\section{Timed Automata Based Fuzzy Cognitive Maps: Basic Concepts}

This section discusses about the methodology, i.e., the Timed Automata based Fuzzy Cognitive Maps (TAFCMs), exploited in this work to simulate a dynamic waste management environment. TAFCMs are new inference engines able to model dynamic changes in cognitive representation of a system and, consequently, perform a more realistic and coherent temporal computation. They achieve this aim by exploiting a synergic approach which combines traditional Fuzzy Cognitive Maps (FCMs) and Timed Automata theory [10. Indeed, a TAFCM can be informally defined as a pair of a timed automaton, used to describe the dynamic evolution of a system, and a FCM, modeling the cognitive behaviour of the system during the first phase of its existence. Therefore, for a complete understanding, it is necessary to know TAFCMs' basic components which, for this reason, will be briefly discussed below before describing TAFCMs in a more detailed way.

\subsection{Basic Components of TAFCMs: Fuzzy Cognitive Maps and Timed Automata}

FCMs are directed graphs with feedback capable of modeling systems through of collection of concepts and causal relations among them. In detail, in the graph, nodes represent concepts, whereas, edges reflect the causal influences among them. The value of a node, contained in the range $[0,1]$, is the degree to which the concept is active in the system at a particular time, whereas, the value of the weights, positioned on the edges and contained in the interval $[-1,1]$, represents the amount of influence existing between two concepts incident on an edge. In particular, the casual relationship between two concepts $C_{i}$ and $C_{j}$ can be: positive which means that an increase in the value of concept $C_{i}$ leads to the increase of the value of concept $C_{j}$, whereas a decrease in the value of concept $C_{i}$ leads to the decrease of the value of concept $C_{j}$; or negative which means 
that an increase in the value of concept $C_{i}$ leads to the decrease of the value of concept $C_{j}$ and vice versa. A graphical representation of a FCM is presented in Fig. 1, Associated with the graphical representation there is a mathematical model that consists of a $1 \times n$ state vector $A$ which includes the values of the $n$ concepts and an $n \times n$ weight matrix $W$ which gathers the weights $W_{i j}$ of the interconnections between the $n$ concepts of the FCM. In this model, the value $A_{i}$ for each concept $C_{i}$ is computed by the following rule:

$$
A_{i}=f\left(\sum_{\substack{j=1 \\ j \neq i}}^{n} A_{j} W_{i j}+A_{i}^{\text {old }}\right),
$$

where $A_{i}$ is the activation level of concept at iteration $t+1, A_{j}$ is the activation level of the concept $C_{j}$ at iteration $t, A_{i}^{\text {old }}$ is the activation level of concept $C_{i}$ at iteration $t$ (it is clear that the variable $t$ just represents the iteration number between two successive FCM matrix computation), and $W_{j i}$ is the weight of the interconnection between $C_{j}$ and $C_{i}$, and $f$ is a threshold function, i.e, a function used to reduce unbounded inputs to a strict range (typically, a sigmoidal function). FCMs have been used in different research [11 and application fields for decision analysis [12] and for distributed cooperative agents [13. However, in spite of their wide applicability, FCMs could be not flexible enough to deal with dynamic systems that change their configurations over time, like social and economical environments and the same urban waste management influenced by government decisions occurring in different and unpredictable moments. Hence, the introduction of TAFCMs capable of enhancing the temporal expressive power of FCMs through the exploitation of a timed automaton.

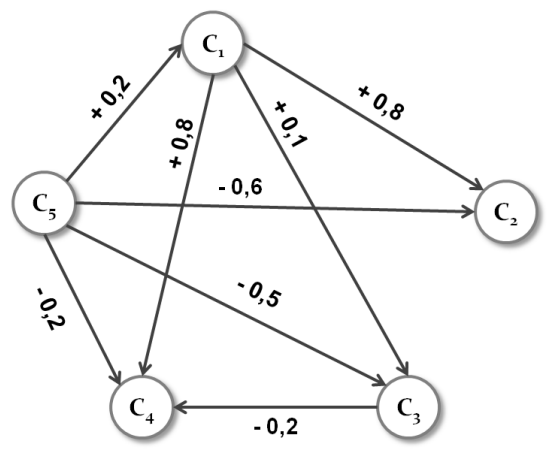

Fig. 1. An example of a simple FCM

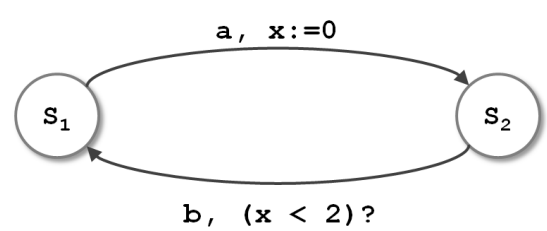

Fig. 2. A Timed Automaton

Timed automata are standard finite-state non-deterministic automata extended with a finite collection of real-valued clocks providing a straightforward way to represent time related events. The transitions of a timed automaton are labeled with a guard (a condition on clocks), an action or symbol on alphabet $\Sigma$, 
and a clock reset (a subset of clocks to be reset). Timed automata start computations with all clocks set to zero and clocks increase simultaneously and uniformly with time while the automaton state is located in a given node. Different from standard non-deterministic machine, transitions in timed automata can be taken if the clocks fulfill the related guards. By taking transition, all clocks belonging to the clock reset collection will be set to zero, whereas the remaining keep their values [14. The transitions occur instantaneously. A simple example [10] of a timed automaton is reported in Fig. 2. The start state of the automaton is $S_{1}$. There is a single clock, named $x$. The annotation $x:=0$ on an edge corresponds to the action of resetting the clock $x$ when the edge is traversed. Instead, the annotation $(x<2)$ ? represents a clock constraint which means that the edge can be traversed only if the value of the clock $x$ is less than 2 . In general, the behavior of the automaton is as follows. The automaton starts in the state $S_{1}$ and moves to state $S_{2}$ reading the symbol input $a$. The clock $x$ gets set to 0 along with this transition. While in state $S_{2}$, the value of the clock $x$ shows the time elapsed since the occurrence of the last symbol $a$. The transition from the state $S_{1}$ to state $S_{2}$ is enabled only if the value of the clock $x$ is less than 2 . The whole cycle repeats when the automaton moves back to state $S_{1}$.

The set of behaviors expressed by a system modeled by means of a timed automaton is defined by a timed language, i.e., a collection of timed words. A timed word is a pair $(\sigma, \tau)$, where $\sigma=\sigma_{1} \sigma_{2} \ldots$ is an infinite word and $\tau=\tau_{1} \tau_{2} \ldots$ is an infinite sequence of time values $\tau_{i} \in \mathbb{R}$ with $\tau_{i}>0$, satisfying constraints of monotonicity and progress (see [10]). In detail, if a timed word $w=(\sigma, \tau)$, it presents the symbol $\sigma_{i}$ at time $\tau_{i}$. Therefore, if $\sigma_{i}$ is an event occurrence then the corresponding component $\tau_{i}$ is interpreted as the time of occurrence of $\sigma_{i}$. As for the timed automaton presented in Fig. 2, the timed language is represented by all words in which $a$ and $b$ alternate and the delay between $a$ and the following $b$ is always less than 2. Therefore, a possible word is $w=(\sigma, \tau)$, where $\sigma=a b a b$ and $\tau=0.0,1.8,2.2,3.7$. Given the word $w=(a, 0.0) \rightarrow(b, 1.8) \rightarrow(a, 2.2) \rightarrow(b, 3.7)$, the automaton starts in the state $S_{1}$ and moves to state $S_{2}$ reading the symbol input $a$ at time 0.0 . The clock $x$ gets set to 0 along with this transition. While in state $S_{2}$, the value of the clock $x$ increases. When the symbol $b$ is read at time 1.8, the automaton moves from the state $S_{2}$ to state $S_{1}$ since the clock $x$ has the value 1.8 which satisfies the guard present on the edge. The automaton moves again to the state $S_{2}$ when the second symbol $a$ is read at time 2.2. Finally, the automaton moves to state $S_{1}$, when the final symbol $b$ is read at time 3.7. Indeed, at time 3.7, the $x$ value is 1.5 , and then, the clock satisfies again the guard.

Formally, this timed behavior is captured by introducing the run concept. Intuitively, a run is a collection of sequential discrete transitions, where each transition denotes an event releasing a task and the guard on the transition (i.e. a temporal constraint) specifies all the possible arriving times of the event. Therefore, by considering the automaton in Fig. 2, the run which corresponds to word $w=(a, 0.0) \rightarrow(b, 1.8) \rightarrow(a, 2.2) \rightarrow(b, 3.7)$ is as follows: 


$$
r:\left\langle S_{1},[0]\right\rangle \underset{0}{\stackrel{a}{\longrightarrow}}\left\langle S_{2},[0]\right\rangle \underset{1.8}{\stackrel{b}{\longrightarrow}}\left\langle S_{1},[1.8]\right\rangle \underset{2.2}{\stackrel{a}{\longrightarrow}}\left\langle S_{2},[0]\right\rangle \underset{3.7}{\stackrel{b}{\longrightarrow}}\left\langle S_{1},[1.5]\right\rangle .
$$

See [10] for more details about the run concept, and, in general, timed automata.

\subsection{TAFCMs: Merging of Timed Automata and Fuzzy Cognitive Maps}

TAFCMs are new timed cognitive inference engines capable of modeling dynamic complex systems. They enhance the modeling power of FCMs by adding three new timing mechanisms, named Top-time (T-Time), cognitive era and cognitive configuration. In detail, the exploitation of these new cognitive concepts enables FCMs to represent a generic system as a biological entity that lives its existence by crossing a sequence of time periods (cognitive eras), each one representing the longest interval time in which the system does not change its cognitive configuration, i.e., its concepts and causal relationships. In order to implement this new mechanism, TAFCMs exploit a timed automaton whose possible behaviors define all the potential sequences of cognitive eras (and the related cognitive configurations) that the system could cover during its life-cycle. More in detail, TAFCMs improve FCMs by associating each state in a timed automaton with a cognitive configuration which describes the behavior of a system in a time interval. Therefore, informally (see 9 for a mathematical definition), a TAFCM has two components: a timed automaton $T_{M}$ that describes the dynamic evolution of a system and a FCM $F^{0}$ modeling the cognitive behavior of the system during first phase of its existence. Once that the automaton computation starts over a given timed word, the state transitions will adaptively modify the initial FCM in order to model time-dependency of the system. In order to transform the structure of a FCM representing a cognitive configuration, the following collection of operators has been introduced:

- To add concepts $(\oplus)$;

- To add causal relationships $(\boxplus) ;$

- To remove concepts $(\ominus)$;

- To remove causal relationships $(\boxminus)$;

- To magnify/reduce the strength of a causal relationships $(\dot{+}$ for an additive modification and $\square$ for a multiplicative modification);

- To magnify/reduce the level of system concept ( $\uparrow$ for an additive modification and $\ddagger$ for a multiplicative modification).

The mapping between the states of timed automaton $T_{M}$ and the collection of cognitive configurations computable starting from $F^{0}$ by applying different sequence of operators is called cognitive evolution, whereas, the progression of the cognitive eras is performed by using the so called cognitive run, i.e., a cognitive extension of the initial idea of the run of a timed automata. As aforesaid, intuitively, a run is a collection of sequential discrete transitions, where each transition denotes an event releasing a task and the guard on the transition (i.e. 
a temporal constraint) specifies all the possible arriving times of the event. In the context of the cognitive representation, the $i^{\text {th }}$ discrete transition can be used to move a system among cognitive eras and release a task changing the cognitive configuration of the system, i.e., transforming the FCM that represents the cognitive configuration during $i^{\text {th }}$ cognitive era into a new one modeling the system during $(i+1)^{t h}$ cognitive era. Both the cognitive evolution and the cognitive run are potentially based on the infinite concept in the system. In fact, the cognitive evolution can exploit an infinite application of cognitive operators to compute the mappings between the automaton states and the FCMs representing cognitive configurations, whereas, the cognitive run uses a timed word, defined as a infinite sequence of ordered pairs, to describe the cognitive/dynamic behavior of the system. Consequently, in order to simulate the behavior of a TAFCM during the first $n$ cognitive eras, the annotation of $n^{t h}$-order cognitive evolution and $n^{\text {th }}$-cognitive run has been introduced.

See [9] for a formal definition of cognitive evolution, cognitive run and T-Time, whereas, our discussion about TAFCMs ends with an example taken by [9]. In this sample, a timed cognitive system describing the behavior of an ecosystem formed by three initial elements, predators $\left(P_{2}\right)$, preys $\left(P_{1}\right)$ and grass for preys $(G))$ is given. Figs. 3 and 4 show, respectively, the initial cognitive configuration $F^{0}$ of proposed system and the modified timed automaton $T_{M}$ that defines the dynamic behavior. In order to show how a TAFCM allows to dynamically modify the cognitive concepts of the system, let us consider, for instance, the transition from the state $s_{0}$ to $s_{2}$. When the automaton traverses this transition, the new concept hunter $(H)$ is added to system to modify the cognitive concepts, $G, P_{1}$ and $P_{2}$ in a novel and unpredicted way. This transformation is denoted by the operator $\oplus$ present on the transition. Moreover, by considering the timed word $w=(\sigma, \tau)=(a, 0.2) \rightarrow(b, 0.4)$, we can compute the $3^{t h}$-order cognitive run $r_{c}^{3}$ (with the clock period $k=0.1$ ) as follows:

$$
r_{c}^{3}:\left\langle s^{0},[0]\right\rangle \underset{0.2}{\stackrel{a, \oplus}{\longrightarrow}}\left\langle s^{1},[0.2]\right\rangle \underset{0.4}{\stackrel{b, \boxplus}{\longrightarrow}}\left\langle s^{2},[0.4]\right\rangle
$$

which defines three cognitive eras, respectively starting at time $0,0.2$ and 0.4 . Fig. 5 shows the three corresponding cognitive configurations.

\section{A TAFCM for Urban Waste Management}

In this section, we show how a TAFCM can be exploited to simulate and evaluate the behavior of a dynamic waste management system. In general, it is the municipality's responsibility to manage waste including its collection, treatment, and safe disposal. During the term of a municipal government, in its effort to improve the waste management, it may make several decisions in order to modify its political plan. Such decisions are very complex due to great number of the involved factors, often in conflict each other, and usually dependent on the time in which they are taken. In particular, this last feature denotes a dynamic nature of urban waste management problem. Therefore, thanks to its ability 


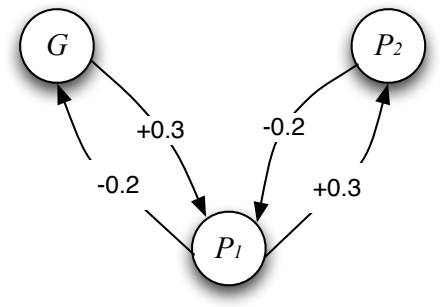

Fig. 3. The initial cognitive configuration of ecosystem sample

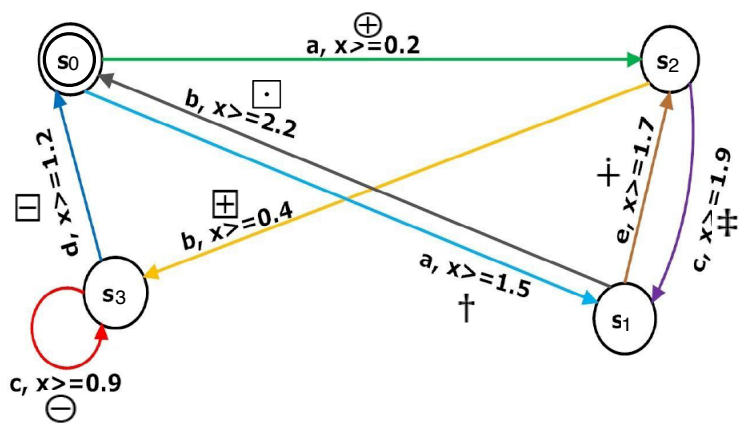

Fig. 4. The cognitive timed automaton that describes the dynamic behavior of the ecosystem sample

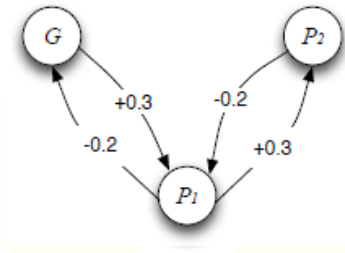

$F^{0}$

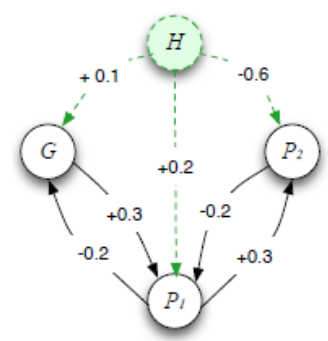

$$
F^{1}=\oplus\left(F^{0}, H=0.8,\right.
$$$$
(H, G)=+0.1
$$$$
\left(H, P_{1}\right)=+0.2
$$$$
\left.\left(H, P_{2}\right)=-0.6\right)
$$

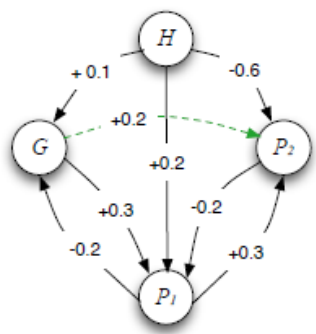

$F^{2}=\boxplus\left(F^{1}\left(G, P_{2}\right)\right)=+0.2$ first cognitive configuration $F^{0}$; the graph (b) shows the cognitive configuration $F^{1}$ related to the state $s_{2}$, obtained by applying the $\oplus$ operator over the FCM $F^{0}$; the graph (c) shows the cognitive configuration $F^{2}$ related to the state $s_{3}$, obtained by applying the $\boxplus$ operator over the FCM $F^{1}$. 
to model systems evolving over time, a TAFCM could represent a promising methodology for efficiently designing an urban waste management simulation system. Our idea is to use a TAFCM for comparing different political strategies so to allow municipality to make the most opportune decisions at beginning of its government mandate.

As aforesaid, factors involved in the urban waste management are a lot, but above all, characterized by different nature: economical, social and environmental. In particular, in this work, we consider the following factors:

- population: this factor refers to number of residents in the city at issue. It is very important to estimate the amount of produced waste;

- migration into city: this factor refers to degree of migration which affects the city. It is relevant over years in order to estimate the increasing of the population;

- modernization: this factor includes all municipality activities aimed at improving life quality of people. For instance, they involve infrastructure and transportation improvements;

- waste per area: this factor refers to average amount of waste per area produced by population. It represents a critical value which should be maintained under control and minimized;

- sanitation facilities: this factor includes all activities of municipality and not only devoted to improve health quality of people.

- disease percentage: this factor refers to number of deseases per 1000 residents. It is a critical value which should be monitored because its increasing may lead to an sanitation alarm;

- bacteria per area: this factor refers to average amount of bacteria per area. It represents a critical value which, obviously, should be minimized in order to improve life quality of people;

- economical funds: they represent the money reserved to urban waste management. Therefore, for instance, they can be used to build new incinerators, to look for location for new landfills, to promote educational plans or recycling programs, to pay workers responsible for collecting waste door to door or cleaning streets. These municipality's funds could be increased by periodical or one-off government payments.

Starting from all involved factors, there are several possible strategies which the municipality could follow. They belong to these four categories:

- waste disposal: it includes all disposal methods such as dumping, landfilling and incineration of waste;

- waste recycling: it refers to recycling of all materials that can be reprocessed either into the same material (closed loop) or a new product (open loop). Therefore, recycling is not only an alternative to disposal, but it allows also the reduction of the amount of virgin materials used for the manufacture of new products;

- waste processing: it includes all activities devoted to the treatment and recovery of materials or energy from waste through thermal, chemical, or biological procedures; 
- waste minimization: it is aimed at decreasing the generation of waste by means of education and optimized production process rather than enhancing technology for handling waste. In detail, minimizing waste production is viewed as result of a maximization of the efficiency of available resources, and as a consequence, it may lead to decrease costs to treat waste to be disposed.

In order to simulate the application of different strategies, a TAFCM composed of the initial FCM $F^{0}$ (see Fig. 6) and the timed automaton $T_{M}$ (see Fig. (7) is introduced. In particular, $F^{0}$ models the collection of concepts related to waste management, before possible strategies are applied. The mapping between the considered factors and the cognitive concepts is as follows: $c_{1} \equiv$ population, $c_{2} \equiv$ migration into city, $c_{3} \equiv$ modernization, $c_{4} \equiv$ waste per area, $c_{5} \equiv$ sanitation facilities, $c_{6} \equiv$ disease percentage, $c_{7} \equiv$ bacteria per area, $c_{8} \equiv$ economical funds. The weights characterizing the relations among concepts had been defined by an expert of domain. Instead, the timed automaton $T_{M}$ allows to model municipality strategies by transforming $F^{0}$ at right opportunities. However, for sake of simplicity, the reported $T_{M}$ models only two possible strategies belonging to the first presented category (see section 4). The timed automaton $T_{M}$ considers only a clock named $x$. Each edge of $T_{M}$ is labeled with a symbol, representing an occurred event, a guard, indicating the temporal constraint which $x$ must satisfy to cross transition and a sequence of transformation operators which allows to opportunely modify the initial FCM. In particular, each operator takes in input concepts which are affected by the change and, according to the kind of operator, a real value representing the amount of modification. In this work, the temporal constraint for cognitive transitions is in the unit of Year, whereas, the simulated time duration is equal to a complete municipality mandate (5 years). However, in spite of the implemented TAFCM, the exploited methodology represents a general framework able to efficiently simulate also other, more expanded, waste management scenarios characterized by a greater number of factors, new strategies and a different temporal constraint.

\section{Experimental Results}

In this section, we demonstrate how a TAFCM can efficiently simulate a dynamic waste management system. Indeed, our experiment will show how a TAFCM can be used to support municipality's decisions by providing a temporal simulation of all involved factors. In particular, our experiment illustrates how the implemented TAFCM to succeed in supporting municipality's decisions by detecting the best strategy (between the considered strategies) to be followed in order to minimize the waste and bacteria per area with a good use of government funds reserved for urban waste management.

In detail, the experiment involves the evaluation of two strategies, named program I and program II. The scenario is the following one. Let us consider a city which is not equipped with technological tools for waste treatment, and hence, the municipality decides to build one of them. Besides, the municipality plans 


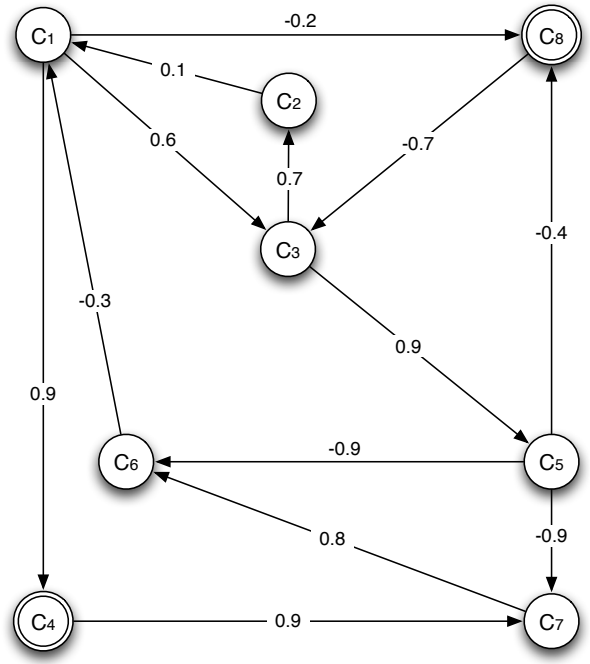

Fig. 6. The cognitive map $F^{0}$

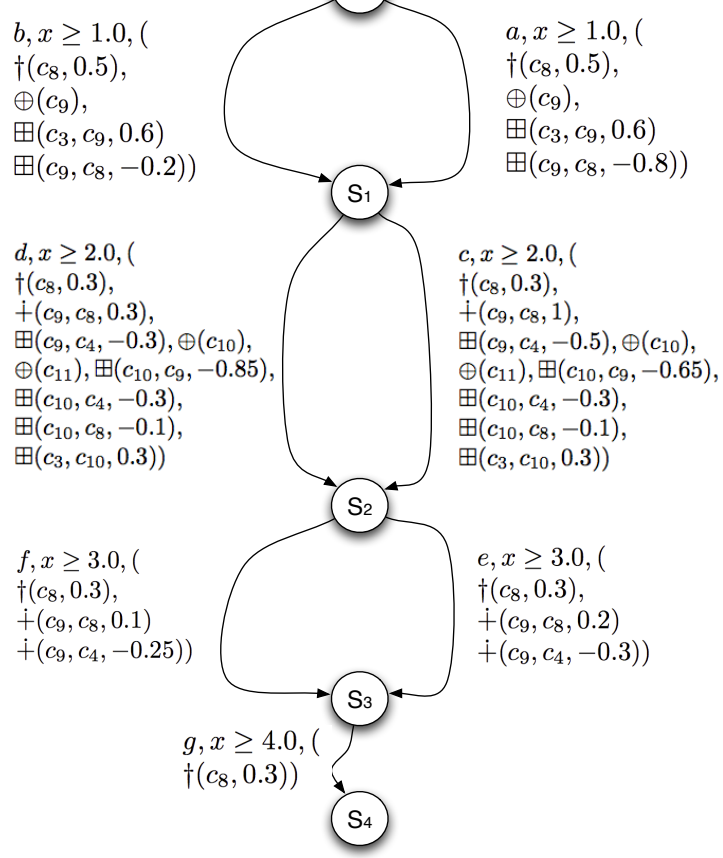

Fig. 7. The cognitive timed automaton $T_{M}$ 
to promote some educational plans in order to teacher people how to minimize the production of waste. The municipality annually receives a fixed amount of economical funds by the central government, and, occasionally, it receives a further economical support. In this scenario, the two considered strategies dissent because of the use of different kinds of technologies:

- program I provides to build high quality and expensive technologies that are characterized by a slow aging and cheap maintenance costs;

- program II provides to build low quality and cheap technologies.

Starting from this description, it is clear the necessity to introduce new factors over time to deal with urban waste management. In detail, they are:

- technology: this factor represents technological tools aiding waste treatment such as incinerators;

- educational plans: this factor refers to a collection of educational programs promoted by the municipality to raise citizen awareness;

- aging: this factor refers to aging which may affect technological tools.

With the help of the implemented TAFCM $\left(F^{0}, T_{M}\right)$, it is possible to decide the best strategy among program I and program II. By taking into account our TAFCM, the two considered strategies correspond, respectively, to two different timed words of the exploited timed automaton $T_{M}$ :

$$
\begin{aligned}
& w_{1}=\left(\sigma_{1}, \tau\right)=(a, 1) \rightarrow(c, 2) \rightarrow(e, 3) \rightarrow(g, 4) \\
& w_{2}=\left(\sigma_{2}, \tau\right)=(b, 1) \rightarrow(d, 2) \rightarrow(f, 3) \rightarrow(g, 4)
\end{aligned}
$$

Both timed words evolve $F^{0}$ through the five years by crossing four cognitive eras whose cognitive configurations are defined, respectively, by cognitive transitions labeled with symbols aceg and bdfg. $w_{1}$ and $w_{2}$ generate a $4^{\text {th }}$-order cognitive evolution with the corresponding $4^{t h}$-order cognitive run which represents the application of opportune decisions in a given time.

Now, let's analyze the effects of application of $w_{1}$ and $w_{2}$ on the initial map $F^{0}$ in order to highlight benefits/drawbacks provided by corresponding strategies. During the cognitive runs related to $w_{1}$ and $w_{2}$, the $\operatorname{TAFCM}\left(F^{0}, T_{M}\right)$ evolves through such events as receiving funds from the central government, improving technologies and educational plans. During their cognitive evolutions, both timed words allow to add new concepts to $F^{0}: c_{9} \equiv$ technology, $c_{10} \equiv$ educational plans, $c_{11} \equiv$ aging.

The whole municipality's behavior with respect to the timed words $w_{1}$ and $w_{2}$ is showed, respectively, in Tables 1 and 2

As highlighted by Figs. 8 and 9, program $I$ associated with $w_{1}$ produces a more effective behavior of the whole municipality decisions because they decrease the value of concepts $c_{4}$ and $c_{7}$, respectively, related to waste per area and bacteria per area, and at the same time, it allows municipality to save economical funds (concept $c_{8}$ ). Therefore, thanks to the exploitation of a TAFCM, it has been possible to identify the best strategy to be followed. 
Table 1. Description of the $4^{t h}$-order Cognitive Evolutions related to timed word $w_{1}$

\begin{tabular}{|c|c|c|}
\hline & Operator & Description \\
\hline I cognitive era & $\begin{array}{l}\oplus\left(c_{9}\right) \\
\dagger\left(c_{8}, 0.5\right) \\
\boxplus\left(c_{3}, c_{9}, 0.6\right) \\
\boxplus\left(c_{9}, c_{8},-0.8\right)\end{array}$ & $\begin{array}{l}\text { High quality (HQ) Technology is added } \\
\text { Municipality increases by } 50 \% \text { its funds } \\
\text { Modernization positively supports } \\
\text { Technology Maintenance }(60 \%) \\
\text { HQ technology reduces by } 80 \% \\
\text { the municipality funds }\end{array}$ \\
\hline II cognitive era & \begin{tabular}{|l|}
$\dagger\left(c_{8}, 0.3\right)$ \\
$\dot{+}\left(c_{9}, c_{8}, 1\right)$ \\
$\boxplus\left(c_{9}, c_{4},-0.5\right)$ \\
$\oplus\left(c_{10}\right)$ \\
$\oplus\left(c_{11}\right)$ \\
$\boxplus\left(c_{11}, c_{9},-0.65\right)$ \\
$\boxplus\left(c_{10}, c_{4},-0.3\right)$ \\
$\boxplus\left(c_{10}, c_{8},-0.1\right)$ \\
$\boxplus\left(c_{3}, c_{10}, 0.3\right)$
\end{tabular} & $\begin{array}{l}\text { Municipality increases by } 30 \% \text { its funds } \\
\text { HQ Technology positively increases } \\
\text { municipality funds } \\
\text { HQ Technology strongly decreases } \\
\text { garbage per area } \\
\text { Municipality starts Educational Plans } \\
\text { Technology aging starts } \\
\text { Educational Plans reduce } \\
\text { waste per area } \\
\text { Educational Plans reduce } \\
\text { municipality funds } \\
\text { Modernization positively influences } \\
\text { Educational Plans }\end{array}$ \\
\hline III cognitive era & $\begin{array}{l}+\left(c_{8}, 0.3\right) \\
\dot{+}\left(c_{9}, c_{8}, 0.2\right) \\
\dot{+}\left(c_{9}, c_{4},-0.3\right)\end{array}$ & $\begin{array}{l}\text { Municipality increases by } 30 \% \text { its funds } \\
\text { HQ Technology positively increases } \\
\text { municipality funds } \\
\text { HQ Technology decreases } \\
\text { waste per area }\end{array}$ \\
\hline IV cognitive era & $\dagger\left(c_{8}, 0.3\right)$ & Municipality increases by $30 \%$ its funds \\
\hline
\end{tabular}

Table 2. Description of the $4^{t h}$-order Cognitive Evolutions related to timed word $w_{2}$

\begin{tabular}{|c|c|c|}
\hline & Operator & Description \\
\hline I cognitive era & $\begin{array}{l}\oplus\left(c_{9}\right) \\
\dagger\left(c_{8}, 0.5\right) \\
\boxplus\left(c_{3}, c_{9}, 0.6\right) \\
\boxplus\left(c_{9}, c_{8},-0.2\right)\end{array}$ & $\begin{array}{l}\text { Low quality (LQ) Technology is added } \\
\text { Municipality increase by } 50 \% \text { its funds } \\
\text { Modernization positively supports } \\
\text { Technology Maintenance }(60 \%) \\
\text { LQ technology reduces by } 20 \% \\
\text { the municipality funds }\end{array}$ \\
\hline II cognitive era & $\begin{array}{l}\dot{\dagger}\left(c_{8}, 0.3\right) \\
\dot{+}\left(c_{9}, c_{8}, 0.3\right) \\
\boxplus\left(c_{9}, c_{4},-0.3\right) \\
\oplus\left(c_{10}\right) \\
\oplus\left(c_{11}\right) \\
\boxplus\left(c_{11}, c_{9},-0.85\right) \\
\boxplus\left(c_{10}, c_{4},-0.3\right) \\
\boxplus\left(c_{10}, c_{8},-0.1\right) \\
\boxplus\left(c_{3}, c_{10}, 0.3\right)\end{array}$ & $\begin{array}{l}\text { Municipality increases by } 30 \% \text { its funds } \\
\text { LQ Technology slowly increases } \\
\text { municipality funds } \\
\text { LQ Technology slowly decreases } \\
\text { waste per area } \\
\text { Municipality starts Educational Plans } \\
\text { Technology aging starts } \\
\text { Educational Plans reduce } \\
\text { waste per area } \\
\text { Educational Plans reduce } \\
\text { municipality funds } \\
\text { Modernization positively influences } \\
\text { Educational Plans }\end{array}$ \\
\hline III cognitive era & $\begin{array}{l}\dot{\dagger}\left(c_{8}, 0.3\right) \\
\dot{+}\left(c_{9}, c_{8}, 0.1\right) \\
\dot{+}\left(c_{9}, c_{4},-0.25\right)\end{array}$ & $\begin{array}{l}\text { Municipality increases by } 30 \% \text { its funds } \\
\text { LQ Technology increases } \\
\text { municipality funds } \\
\text { LQ Technology decreases } \\
\text { garbage per area }\end{array}$ \\
\hline IV cognitive era & $\dagger\left(c_{8}, 0.3\right)$ & Municipality increases by $30 \%$ its funds \\
\hline
\end{tabular}




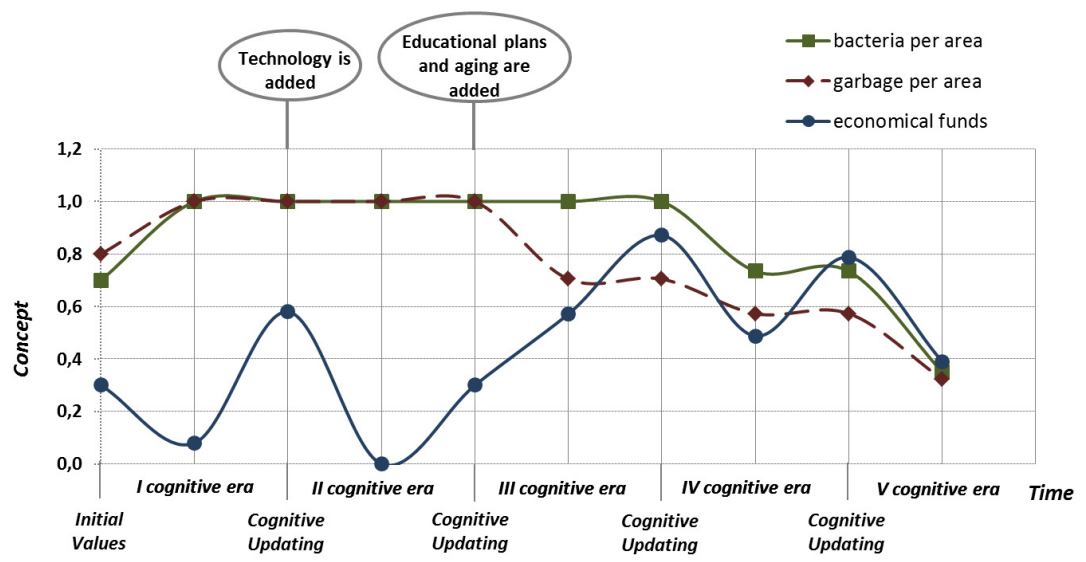

Fig. 8. Concept values $\left(c_{4}, c_{8}, c_{7}\right)$ evolution related to $4^{\text {th }}$-order cognitive run of $w_{1}$

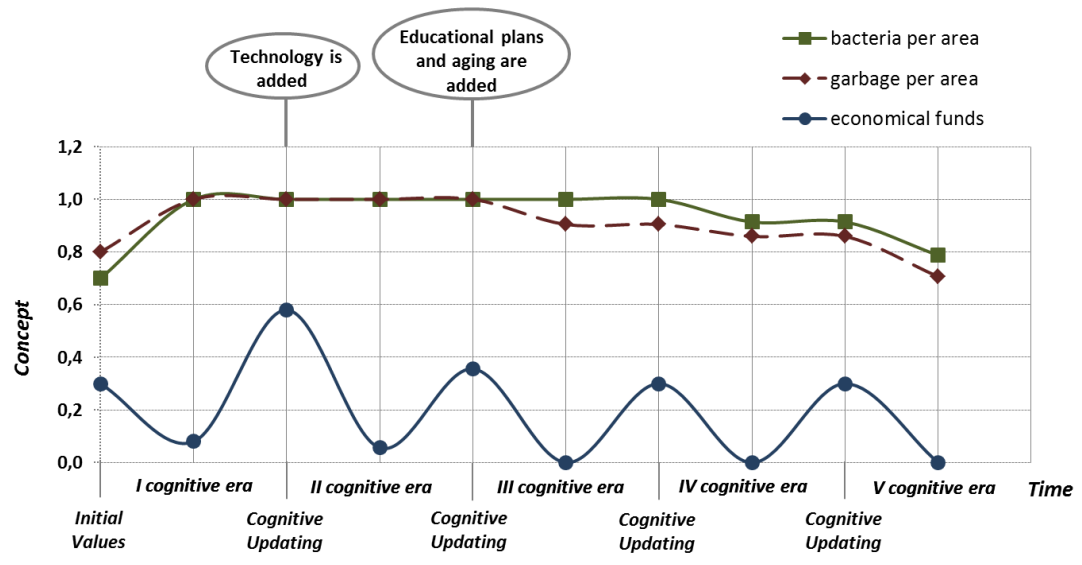

Fig. 9. Concept values $\left(c_{4}, c_{8}, c_{7}\right)$ evolution related to $4^{\text {th }}$-order cognitive run of $w_{2}$

\section{Conclusions}

The management of hazardous and not waste is a significant problem due to socio-economical and environmental impact and the relative homeland defense issues. This paper presents a new method to simulate a dynamic waste management system able to support municipality's decisions by identifying the best strategies to be followed. In particular, the exploited methodology, named Timed Automata based Fuzzy Cognitive maps (TAFCMs), is capable of efficiently managing the waste production problem thanks to its cognitive approach. In detail, TAFCMs allow to model complex systems characterized by a temporal uncertainty, like urban environments, through the exploitation of a double-layered 
temporal granularity based on the concepts of cognitive configuration, cognitive era and cognitive run. As shown by an experiment, TAFCMs are a promising framework for simulating a dynamic waste management system.

However, TAFCMs introduced in this work exploit expert knowledge to design the collection of cognitive eras and configurations representing the temporal behavior of the system under design. Therefore, in the future, our idea is to investigate some machine learning approaches such as evolutionary computation to automatically find suitable cognitives era and configurations for improving the system behavior.

\section{References}

1. Karadimas, N.V., Orsoni, A., Loumos, V.: Municipal Solid Waste Generation Modelling Based on Fuzzy Logic. In: ECMS 2006 - Proceedings of the 20th European Conference on Modelling and Simulation, pp. 309-314 (2006)

2. Karadimas, N.V., Kouzas, G., Anagnostopoulos, I., Loumos, V.: Urban Solid Waste Collection and Routing: the Ant Colony Strategic Approach. International Journal of Simulation: Systems, Science and Technology 6, 45-53 (2005)

3. Gnoni, M.G., Mummolo, G., Ranieri, L.: A mixed-integer linear programming model for optimisation of organics management in an integrated solid waste system. Journal of Environmental Planning and Management 6(51), 833-845 (2008)

4. Hsieh, H.N., Ho, K.H.: Optimization of solid waste disposal system by linear programming technique. J. Resour. Management Technology 4(21), 194-201 (1993)

5. Lund, J.R., Tchobanoglous, G.: Linear programming for analysis of material recovery facilities. J. Environ. Eng., ASCE 5(120), 1093-1095 (1994)

6. Jin, L.: A type-II fuzzy optimization model for municipal solid waste management. In: 2011 Eighth International Conference on Fuzzy Systems and Knowledge Discovery (FSKD), pp. 55-59 (2011)

7. Huang, G.H., Baetz, B.W., Patry, G.G.: A grey integer programming for solid waste management planning under uncertainty. European J. Oper. Res. 83, 594$620(1995)$

8. Chang, N.B., Wang, S.F.: A fuzzy goal programming approach for the optimal planning of metropolitan solid waste management systems. European J. Oper. Res. 99, 287-303 (1997)

9. Acampora, G., Loia, V.: On the Temporal Granularity in Fuzzy Cognitive Maps. IEEE Transactions on Fuzzy Systems 19, 1040-1057 (2011)

10. Alur, R.: A theory of timed automata. Theoretical Computer Science 126, 183-235 (1994)

11. Miao, Y., Liu, Z.-Q.: On causal inference in fuzzy cognitive maps. IEEE Transactions on Fuzzy Systems 1(8), 107-119 (2000)

12. Zhang, W.R., Chen, S.S., Bezdek, J.C.: Pool2: a generic system for cognitive map development and decision analysis. IEEE Transactions on Systems, Man and Cybernetics 1(19), 31-39 (1989)

13. Zhang, W.-R., Chen, S.-S., Wang, W., King, R.S.: A cognitive-map-based approach to the coordination of distributed cooperative agents. IEEE Transactions on Systems, Man and Cybernetics 1(22), 103-114 (1992)

14. Ericsson, C., Wall, A., Yi, W.: Timed automata as task models for event-driven systems. In: Proceedings of Nordic Workshop on Programming Theory (1998) 